\section{Incidental learning of words used in a memory scanning task}

\author{
D. J. MacKENZIE and R. C. ATKINSON \\ Stanford University, Stanford, Calif. 94305
}

Incidental learning data was obtained by testing if Ss recognized words previously used in a memory scanning task. In the memory scanning task, Ss had compared probe words to memory sets consisting of one to four words, responding positively if the probes matched one of the memory set words or otherwise responding negatively. The incidental learning scores differentiated between certain memory set conditions: (1) Words from memory sets of one had higher scores than those from larger memory sets. (2) Positive probe words were better recognized than all other words. (3) There were no differences between memory sets larger than one item.

How humans retrieve information from short-term memory (STM) is a question that has produced extensive research. Stermberg (1966) reported a memory scanning paradigm in which a $\mathrm{S}$ compared a single digit (probe item) against a set of digits stored in STM (memory set items). The $\mathrm{S}$ made a preassigned positive response if the probe matched an item in the memory set; otherwise, $S$ made a negative response. Sternberg (1966) measured the reaction times (RT) to make positive and negative responses. He found that RT increased linearly with increasing memory set size and that slopes of the linear functions were the same for positive and negative trials. Sternberg (1966) interpreted his data as meaning that $S$ compared the probe items against each member of the memory set serially and exhaustively on both positive and negative trials.

Juola \& Atkinson (1971) have replicated Sternberg's experiment, with the modification that words instead of digits were used. Furthermore, none of the words were ever repeated throughout the experiment. Their $R T$ results were similar to those obtained by Sternberg (1966). The use of words in the memory scanning task has the additional advantage that once the memory scanning trials have been completed, a test can be made for S's incidental memory of the words. It is quite possible that variables affecting RTs in the scanning task might influence information retention in long-term memory (LTM). Thus, the addition of a retention test following a short-term scanning RT study might well provide a two-pronged approach towards the understanding of STM processes. The important question is whether Ss will score high enough on a long-term retention test to justify the making of inferences about the STM processes involved.

If $S$ expects that he will later be tested on his memory for the words, the four alternatives. the memory scanning results might be affected, resulting in $\mathrm{RT}$ data unlike that normally reported. Hence, S should initially be informed only about the memory scanning task. After the RT data is collected, then S can be tested on the information that he may have retained from the memory scanning task. In the experiment to be reported here, the retention test consisted of a four-alternative forced-choice recognition test; each word from the scanning task was presented with three new words, and $S$ had to decide among

Sternberg's (1966) interpretation of his RT data has certain implications

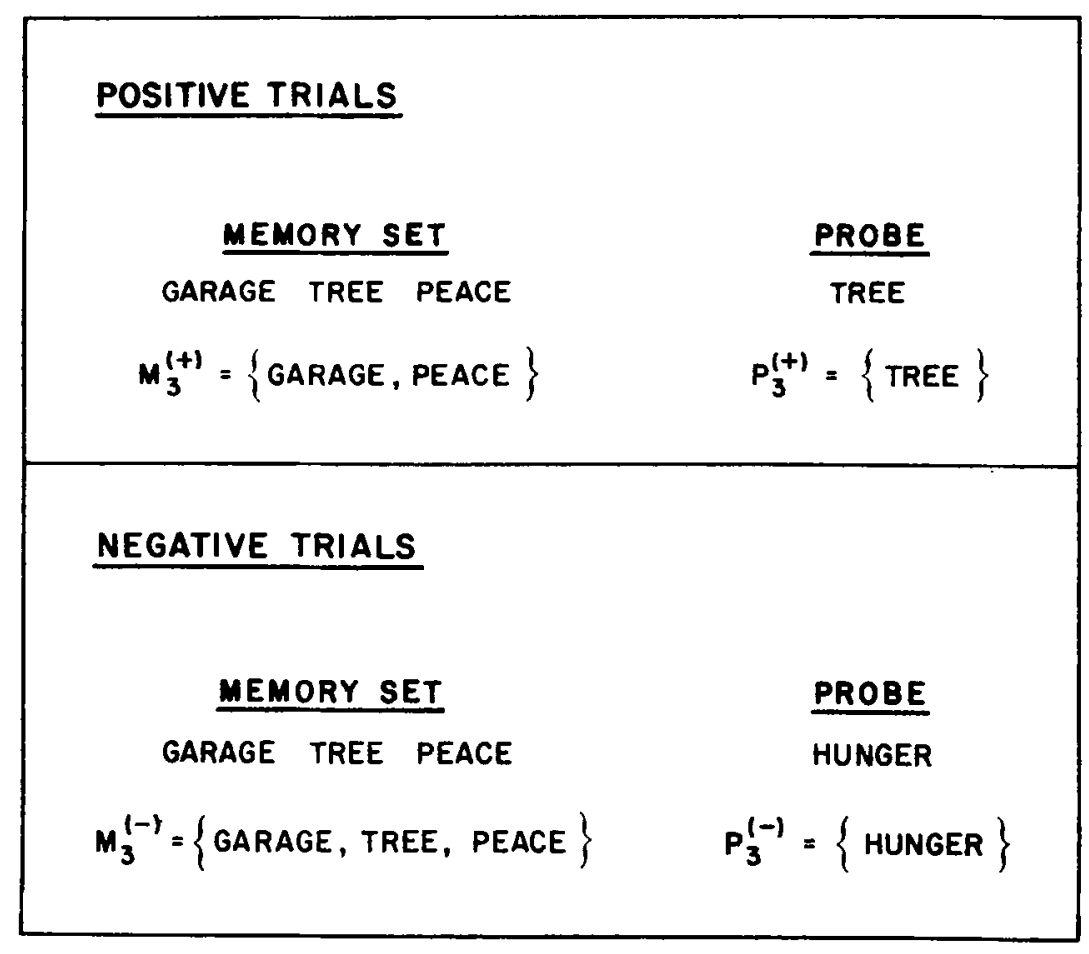

Fig. 1. The notation used to refer to items in the various memory scanning conditions, shown by use of a sample memory set. that can be examined in light of the incidental learning data. The memory scanning conditions under which words are presented should be defined before these implications are made explicit. Figure 1 illustrates the four conditions under which words were scanned. On positive trials, words appearing only in the memory set (GARAGE, PEACE) are denoted $\mathrm{M}_{\mathrm{i}}(+)$ items; for memory sets of one item, the $M_{1}(+)$ condition is undefined. On positive trials, the word used as a probe is also in the memory set and will be designated as $\mathbf{P}_{\mathrm{i}}(+)$ (TREE). On negative trials, all the words in the memory set (GARAGE, TREE, PEACE) will be referred to as $M_{i}(-)$ items. Probe items on negative trials (HUNGER) will be called $P_{i}(-)$ items. The subscript $i$ denotes the size of the memory set; in the above example, the value of $i$ is 3 .

Memory set size, which influences RT in the scanning task, might well have an effect on the long-term retention of information about words used in the memory scanning task. Furthermore, the incidental learning results might vary according to the memory scanning condition in which words were originally presented. One can postulate that the larger the memory set on a given trial, the longer the probe remains in STM, since it must be compared with the various memory set items. If it is further assumed that the longer an item 


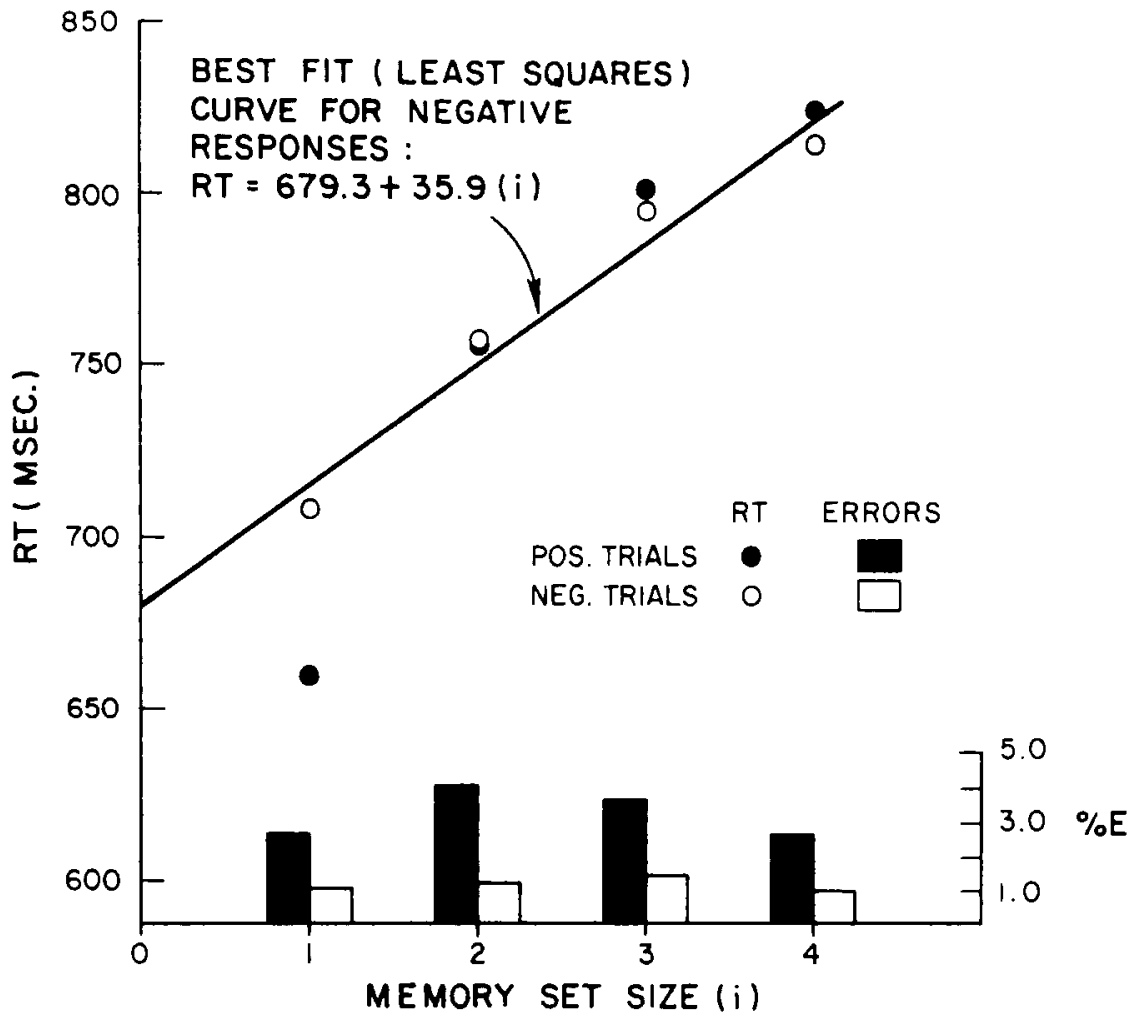

Fig. 2. Mean reaction time for positive and negative responses as a function of memory set size (i). The percentages of trials on which errors occurred are shown at the bottom of the figure.

resides in STM, the better it will be remembered, then certain predictions follow. Namely, as the memory set size $\mathrm{i}$ increases, both $P_{i}(+)$ and $P_{i}(-)$ items should have higher scores on the incidental learning test. Since $\mathbf{M}_{\mathrm{i}}(+)$ and $\mathrm{M}_{\mathrm{i}}(-)$ items are each compared only once against $P_{i}(+)$ and $P_{i}(-)$ items, respectively, the incidental learning functions for $\mathrm{M}_{\mathrm{i}}(+)$ and $\mathrm{M}_{\mathrm{i}}(-)$ should be a constant as $i$ increases.

An alternative prediction for the incidental learning results can be inferred from data on free recall tasks with varying list lengths. In these studies, the probability of recall for a given item decreases as the list length increases. The increase in list length in free recall studies could be regarded as similar to the increase in memory set size that occurs in the memory scanning task. By analogy, one can predict that as memory set size increases, the incidental learning test results for each of the scanning conditions should decrease.

Although no attempt is being made to exhaust the possible predictions for the incidental learning results, a third possibility is worth noting. As memory set size increases, the incidental learning results might reflect both the benefit of more comparisons and the interference of longer list lengths. If both factors-comparison boosts and for 0.5 sec. (4) The $S$ made a positive or negative response by depressing one of two possible keys. (5) After a short interval, the next trial began. The $S$ was instructed to avoid making errors.

The memory scanning task consisted of 128 trials, structured into four consecutive blocks of 32 trials. A single block contained 16 positive and 16 negative trials. There was an equal number of each memory set size and at least one positive probe for each serial position. Within a block, trials were randomly ordered. Between Ss, the blocks were presented in four different orders: each block appeared in the first, second, third, and fourth positions an equal number of times across all Ss. A given word was used on only one scanning trial and was not repeated during the remaining trials.

After the last trial, $\mathrm{S}$ counted backwards by threes from a three-digit number for $1 \mathrm{~min}$. Then $\mathrm{S}$ was given the first indication that she would be required to complete a "memory test" on the words presented during the experiment. The memory test was a recognition test consisting of 384 sets of four words. One word of each set, the target word, had been used in the scanning task; the other three distractor words had not been presented previously. The distractors were 1,152 words selected from Thorndike \& Lorge (1944) to have an equivalent distribution as the target set in frequency and number of syllables. One-fourth of the words from each block and condition of the scanning task were distributed randomly over each fourth of the recognition test. The four-word groups were presented in a seven-page booklet. The $\mathbf{S}$ was asked to underline the target word in each group and to respond even if she had to guess.

The 384 words used in the memory scanning task were selected from the Paivio, Yuille, \& Madigan (1968) norms. The words were divided into eight groups, depending on whether they were "high" or "low" on each of three dimensions: Thorndike-Lorge frequency (F), imagery values (I), and number of syllables (N). For F, "high" meant 50 or more per million, "low" meant less than 50 million. For I, "high" meant 6.00 or greater on the imagery scale, "low" meant less than 6.00. For N, "high" meant two syllables, "low" meant one syllable. Words from each group were evenly distributed over the different conditions of the memory scanning task.

\section{RESULTS}

Reaction times from the memory scanning task are shown in Fig. 2. As in the Juola \& Atkinson (1971) study, the negative RT curve appeared to be a linear function of memory set size and 


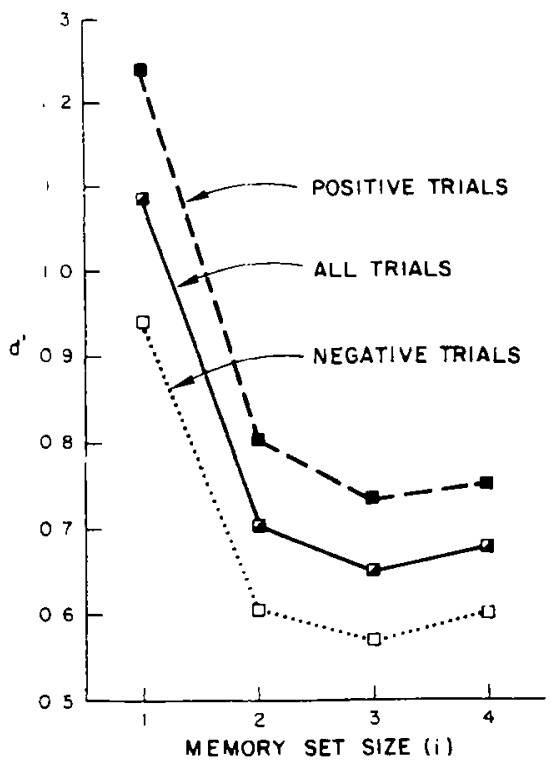

Fig. 3. Mean $d^{\prime}$ on the recognition test for words from the memory scanning task as a function of memory set size (i).

a straight line was fit to the data using the method of least squares. That the slope and intercept ( 35.9 and 679.3 , respectively) of the estimated line in this study were larger than those reported by Juola \& Atkinson (1971) (26.3 and 617 , respectively) was probably due to the different criteria for including trials in the RT data. Since words from each block of the scanning task were included in the incidental learning test of this study, trials from all the blocks were used to compute RTs. In the Juola \& Atkinson (1971) study, the first block of trials was regarded as practice and was omitted from the RT data.

Except for the memory sets with one item, positive RTs were similar to the negative RTs. The positive RT for a memory set of one was faster than the predicted RT from the best-fitting straight line, a result also observed in the Juola \& Atkinson (1971) experiment. The overall error rate was $2.2 \%$. More errors occurred on positive trials than on negative trials [ $3.2 \%$ vs $1.2 \%, t(35)=4.22, p<.001]$.

The probability correct on the recognition test averaged over all words, uncorrected for guessing, was .473. This probability indicates that $\mathrm{Ss}$ retained a significant amount of information about the words used in the memory scanning task. To correct for guessing, the percent correct scores on the incidental learning test for each $S$ and each condition were transformed to $d^{\prime}$ scores. The $d^{\prime}$ values were computed under the assumptions of signal detection theory when applied to a four-alternative forced-choice test; see Green \& Swets (1966) for a discussion of the $d^{\prime}$ transformation.

The solid line in Fig. 3 represents the $d^{\prime}$ values averaged over all items in each memory set size, combined across positive and negative trials and across probed and nonprobed words. The d' value for memory sets of one item was significantly greater than the $d^{\prime}$ values for larger memory sets, all of which appeared approximately equal. When the probed and nonprobed memory set items are separated into positive and negative trials, the $d^{\prime}$ values on positive trials appeared to be greater than on negative trials. Table 1 indicates that the difference between $d^{\prime} s$ in Fig. 3 for positive and negative trial memory sets was due to the relatively high $d^{\prime}$ values for $P_{i}(+)$ items. For a memory set of one, there are no nonprobed items on positive trials, and consequently, it is not possible to compare $M_{1}(+)$ and $M_{1}(-)$ items. For larger memory set sizes, Table 1 shows that $M_{i}(+)$ and $M_{i}(-)$ items had very similar mean $d^{\prime}$ values (.58 and .60, respectively), and as memory set size increased, d' values remained virtually constant. For a memory set of one, however, the d' for $M_{1}(-)$ items is significantly greater than for larger memory sets. There were no other significant differences among $d^{\prime}$ for a given scanning condition as memory set size varied.

For memory sets of four items, d' values for each serial position were computed for each of the scanning conditions. There were no significant serial position effects. Values of $d^{\prime}$ were also computed for the three dimensions along which the sample of words was categorized. Low-frequency words were better remembered than high-frequency words $(.81$ to .64 , $\mathrm{p}<.01)$. Two-syllable words had larger $\mathrm{d}^{\prime}$ values than one-syllable words (.79 to $.65, \mathrm{p}<.01)$. There was no difference between high- and low-imagery words (.72 to .73 ).

\section{DISCUSSION}

The principal result from this experiment was that $S$ s do retain a significant amount of information about the memory scanning task. The information retained can be considered to be incidentally learned because $S s$ had no knowledge or expectation that the words used in the nemory scanning task would be tested later. Other results were: (1) Memory set items that were probed were better recognized than the other memory set items. (2) Except for memory sets of size one, untested memory set items from positive trials were recognized to the same extent as those from negative trials. (3) On negative trials, nonprobed items from a memory set of one were better recognized than nonprobed items from larger memory sets.

The d' values for $P_{i}(+), M_{i}(+)$, and $M_{i}(-)$ items suggested that the probing of one item in a memory set increased the probability of recognizing that item without affecting the untested items. The functions relating $\mathrm{d}^{\prime}$ scores for the various scanning conditions to memory set size were flat. Neither $P_{i}(+)$ nor $P_{i}(-)$ items showed any evidence that $d$ ' increased as memory set increased, a result that would be expected if comparisons during scanning tended to boost recognition probability. Nor was the overall decrease for all scanning conditions as memory set increased evident in any of the functions. 'The third possible outcome discussed earlier was that $P_{i}(+)$ and $P_{i}(-)$ items might be affected by both the boost due to added comparisons and the interference due to list length. The relative contribution of the comparison and list length effects would determine the $d^{\prime}$ function as memory set size increased. If these effects cancelled each other, a flat function of $\mathrm{d}^{\prime}$ would be expected as memory set size increased. The $\mathrm{P}_{\mathrm{i}}(+)$ and $P_{i}(-)$ curves were flat and did fit a mixed model. However, this mixed model also predicted decreasing $\mathrm{M}_{\mathrm{i}}(+)$ and $\mathrm{M}_{\mathrm{i}}(-)$ functions, since such items would be affected by the list length interference result; such functions were not observed. Finally, it was not

Table 1

Mean $d^{\prime}$ and SD Compared Within Memory Scanning Conditions Across Memory Set Size

\begin{tabular}{|c|c|c|c|c|c|c|c|}
\hline \multirow{2}{*}{$\begin{array}{l}\text { Scanning } \\
\text { Condition }\end{array}$} & & \multicolumn{4}{|c|}{ Memory Set Size } & \multirow[b]{2}{*}{ Overall } & \multirow{2}{*}{$\begin{array}{c}\text { LSD. } \\
(\mathrm{p}<.05)\end{array}$} \\
\hline & & 1 & 2 & 3 & 4 & & \\
\hline $\mathbf{p}_{\mathrm{i}}^{(+)}$ & $\begin{array}{l}\text { Mean } \\
\text { SD }\end{array}$ & $\begin{array}{r}1.24 \\
.76\end{array}$ & $\begin{array}{r}1.12 \\
.55\end{array}$ & $\begin{array}{r}1.01 \\
.58\end{array}$ & $\begin{array}{r}1.07 \\
.53\end{array}$ & $\begin{array}{l}1.11 \\
1.22\end{array}$ & .42 \\
\hline $\mathbf{M}_{\mathbf{i}}^{(+)}$ & $\begin{array}{l}\text { Mean } \\
\text { SD }\end{array}$ & - & $\begin{array}{l}.49 \\
.46\end{array}$ & $\begin{array}{l}.60 \\
.40\end{array}$ & $\begin{array}{l}.65 \\
.31\end{array}$ & $\begin{array}{l}.58 \\
.68\end{array}$ & .23 \\
\hline $\mathrm{M}_{\mathrm{i}}{ }^{(-)}$ & $\begin{array}{l}\text { Mean } \\
\text { SD }\end{array}$ & $\begin{array}{l}.94 \\
.60\end{array}$ & $\begin{array}{l}.61 \\
.39\end{array}$ & $\begin{array}{l}.57 \\
.35\end{array}$ & $\begin{array}{l}.61 \\
.31\end{array}$ & $\begin{array}{l}.68 \\
.86\end{array}$ & .29 \\
\hline$P_{i}(-)$ & $\begin{array}{l}\text { Mean } \\
\text { SD }\end{array}$ & $\begin{array}{l}.81 \\
.53\end{array}$ & $\begin{array}{l}.77 \\
.51\end{array}$ & $\begin{array}{l}.87 \\
.58\end{array}$ & $\begin{array}{l}.69 \\
.58\end{array}$ & $\begin{array}{r}.79 \\
1.10\end{array}$ & .38 \\
\hline
\end{tabular}

Note-L.SD = least significent difference 
predicted by any of these notions that $M_{1}(-)$ items would be better recognized than $M_{2}(-), M_{3}(-)$, and $\mathrm{M}_{4}(-)$ items.

The elevation of the $d^{\prime}$ values for $M_{1}$ (-) items was one of the few significant differences found in the incidental learning data. The recognition data was mirrored by a decrease in RT for memory sets of one item for positive responses. Possibly, the peak at $M_{1}(-)$ was caused by a quantitative difference in the length of time certain operations were performed on these words. One explanation of the fast $\mathrm{RT}$ for the single item memory set hinges on the hypothesis that before the probe was presented, memory set items were stored in a buffer in STM. Before they could be compared to the probe, they had to be switched to a "comparator" stage. In such a system, memory sets of one item could be transferred to the comparator before the probe was presented. Items in larger memory sets remained in the buffer until the probe appeared and then were transferred one by one into the comparator. Otherwise, under these assumptions, the actual comparison process per item lasted the same length of time regardless of memory set size, except for $M_{1}(-)$ items. The RT data indicated that only the positive trials produced the fast $R T$ which is assumed to result when memory sets of a single item enter the comparator stage at an earlier point in the scanning process than items from larger memory sets. Memory sets of one item on negative trials presumably enter the comparator stage at the same early point as on positive trials, but the RT for $M_{1}(-)$ items does not reflect this advantage. It follows that $M_{1}(-)$ items remain longer in the comparator stage and therefore are better learned by $S$.
This argument cannot be correct. The comparator notion again would predict that $\mathrm{P}_{\mathrm{i}}(+)$ and $\mathrm{P}_{\mathrm{i}}(-)$ items should have increasing $d^{\prime}$ values as memory set size increased-a result not obtained in this experiment.

\section{REFERENCES}

GREEN, D. \& SWETS, J. A. Signal detection theory and psychophysics. New York: Wiley, 1966.

JUOLA J. F.. \& ATKINSON, R. C. Memory scanning for words versus categories. Journal of Verbal Learning \& categones. Journal of Verbal Learnin

PAIVIO. A.. YUILLE, J. C.. \& MADIGAN, $S$. Concreteness, imagery, and meaningfulress values for 925 nouns. Journal of Experimental Psychology, 1968, 76. (1, Pt. 2), 1-25.

STERNBERG, S. High-speed scanning in human memory. Science, 1966. 153. 652-654.

THORNDIKE, E. L.. \& LORGE, I. The leacher's word book of 30,000 words. New York: Bureau of Publications. Teachers College, Columbia University. 1944. 